\title{
Effects of the Cold Pressor Test on Cardiac Autonomic Control in Normal Subjects
}

\author{
L. MOUROT ${ }^{1}$, M. BOUHADDI ${ }^{1,2}$, J. REGNARD $^{1,2}$ \\ ${ }^{1}$ Université de Franche Comté, EA 3920 and IFR133, Physiologie, Faculté de Médecine and ${ }^{2}$ Centre \\ Hospitalier Universitaire, Explorations Fonctionnelles - Physiologie, Besançon, France
}

Received July 24, 2007

Accepted November 27, 2007

On-line January 17, 2008

\begin{abstract}
Summary
The cold pressor test (CPT) triggers in healthy subjects a vascular sympathetic activation and an increase in blood pressure. The heart rate (HR) response to this test is less well defined, with a high inter-individual variability. We used traditional spectral analysis together with the non-linear detrended fluctuation analysis to study the autonomic control of HR during a 3-min CPT. 39 healthy young subjects $(23.7 \pm 3.2$ years, height $180.4 \pm 4.7 \mathrm{~cm}$ and weight $73.3 \pm 6.4 \mathrm{~kg}$ ) were divided into two groups according to their HR responses to CPT. Twenty subjects have a sustained increase in HR throughout the test with reciprocal autonomic interaction, i.e. increase in sympathetic activity and decrease vagal outflow. In the 19 remainders, HR decreased after an initial increase, with indication of involvement of both sympathetic and vagal outflow. Baseline evaluation of the subjects revealed no difference between the two groups. Nevertheless, a higher sympathetic activity at the skin level during CPT was present in the group with decreased HR. Further studies are needed to explain why healthy subjects react differently to the CPT and if this has potential clinical implications.
\end{abstract}

\section{Key words}

Heart rate variability $\bullet$ Spectral analysis $\bullet$ Detrended fluctuation analysis $\bullet$ Fractal $\bullet$ Cold pressor test

\section{Corresponding author}

L. Mourot, Laboratoire de Physiologie, Faculté de Médecine et de Pharmacie, F-25030 Besançon Cedex FRANCE. Fax: +33 3.81.66.56.14. E-mail: mourotlaurent@hotmail.com

\section{Introduction}

The cold pressor test (CPT) is typically performed by immersing a subject's hand into ice water $\left(1-5{ }^{\circ} \mathrm{C}\right)$ for a short period of time (1-6 min) while measuring blood pressure (BP) and heart rate (HR). In normal subjects, a vascular sympathetic response increased peripheral resistance and a sustained increased BP is observed (Victor et al. 1987, Fagius et al. 1989, Weise et al. 1993, Stančák et al. 1996, Sendowski et al. 2000, Cui et al. 2002).

The HR response is less well defined, more variable on an individual basis (Jauregui-Renaud et al. 2001, Glenn and Ditto 2004) and not homogeneous for the entire CPT period. Two major patterns could be distinguished with either an increased (LeBlanc et al. 1975, Shibahara et al. 1996, Jauregui-Renaud et al. 2001, Dishman et al. 2003) or an unchanged HR (Weise et al. 1993, Sendowski et al. 1997, Cui et al. 2002, Fu et al. 2002). The latter response appeared less to be a "true" unchanged HR than a biphasic alteration, with an initial increase followed by a slow decrease that could return toward the control values (Victor et al. 1987, Stančák et al. 1996, Sendowski et al. 1997, Cui et al. 2002). Contrary to the vascular control, the autonomic HR control needs to be precise during CPT. Initially, a decrease in cardiac vagal outflow was accepted together with the sympathetic involvement (Frey et al. 1980a, Dishman et al. 2003, Tulppo et al. 2005, Wirch et al. 2006). However, these changes were not always found. An increase in vagal activity (Frey et al. 1980b, Shibahara et al. 1996, Sendowski et al. 1997, Glenn and Ditto 2004) induced by baroreceptor activity stimulation has also been hypothesized. This vagal stimulation should occur concomitantly to the persistent sympathetic involvement leading to cardiac autonomic 
co-activation (Weise et al. 1993). However, to our knowledge, this was not verified, mainly because in these previous reports the cardiac autonomic control was studied by spectral analysis of heart rate variability (HRV). However, this method may not offer a proper assessment of the HR dynamics during CPT because of limitations inherent in its stationary hypothesis (Task Force 1996).

New analysis techniques, such as analysis of fractal scaling exponents by detrended fluctuation analysis (DFA), have been developed to probe features in HRV that are not detectable by traditional analysis method. With DFA, the short-term (from 4 to 11 beats) fractal organization in human HRV is expressed as a scaling exponent named $\alpha_{1}$. Changes in $\alpha_{1}$ allowed highlighting of cardiac autonomic co-activation or reciprocal changes in vagal/sympathetic activity (Tulppo et al. 2001a, 2005). Therefore, DFA may help to describe the cardiac autonomic status during CPT.

The aim of the present research was to study the autonomic control of HR during CPT. For that purpose, the subjects were divided into two groups according to their HR response, and their autonomic control profiles were studied by means of linear and non-linear HRV analysis. We hypothesized that in the group with sustained increased HR, $\alpha_{1}$ would increased, suggesting a decrease in cardiac vagal outflow together with sympathetic activation, while in the group with an increase followed by a decrease in HR, $\alpha_{1}$ would decrease suggesting cardiac autonomic co-activation. Differences in baseline autonomic characteristics were suspected to explain these two HR responses.

\section{Methods}

\section{Subjects}

Forty male students (age 23.6 \pm 3.2 years mean \pm $\mathrm{SD}$; height $180.4 \pm 4.6 \mathrm{~cm}$; and weight $73.4 \pm 6.4 \mathrm{~kg}$ ) voluntarily participated in the study. That was the first time that they have to perform a cold pressor test. Their medical history and a medical examination were used to exclude subjects with cardiovascular, pulmonary, or metabolic diseases. The subjects were normotensive and none was taking any medication. The study protocol complied with the Helsinki declaration for human experimentation. The subjects were informed of the organization and details of the study and signed an informed consent form, which was approved by our local ethics committee.

\section{Testing protocol}

The subjects were instructed to fast for at least $3 \mathrm{~h}$ before testing, and were asked to refrain from ingesting beverages containing caffeine and alcohol and not to exercise during the $24 \mathrm{~h}$ preceding each test. They were studied in the supine position in a quiet, dimly lighted room (ambient temperature, 26-27 ${ }^{\circ} \mathrm{C}$ ). Before each test, the subjects, wearing short pants and T-shirts, rested $20 \mathrm{~min}$ to ensure hemodynamic stabilization. Then, baseline measurements started for $10 \mathrm{~min}$. Subsequently, the subject immersed its left hand to the wrist into a $0-1{ }^{\circ} \mathrm{C}$ water bath for a period of $3 \mathrm{~min}$, followed by removal of the hand from the bath and continuation of recording for another $5 \mathrm{~min}$.

According to their HR response to the CPT, the subjects were divided into two groups. One group (CPTi) was constituted with subjects that react to the test with an increase in HR, i.e. first an increase in HR and then either a further increase or a maintenance of HR until the end of the test. The second group (CPTd) was constituted with subjects that react to the test with an initial increase followed by a decrease in HR of more than 5 beats per min (mean over $10 \mathrm{~s}$ ) compared to the peak HR achieved during the test. If HR decreased less than 5 beats, the HR was considered to be maintained (Fig. 1).

\section{Hemodynamic measurements}

Beat-to-beat BP was measured continuously using the Finometer ${ }^{\circledR}$ (Finapres Medical System, Amsterdam, The Netherlands). This device measures arterial pressure through a cuff wrapped around the middle phalanx of the middle finger. It has been demonstrated that finger BP recordings can accurately reproduce the beat-to-beat changes in intra-arterial blood pressure induced by a cold pressor stimulus (Parati et al. 1989). Arterial pulse pressure (PP, mmHg) was calculated from systolic (SBP) minus diastolic (DBP) blood pressure. The arterial pressure signal was analyzed using the Beatscope Software (TNO-TPD, Biomedical Instrumentation). Heart rate (HR) was derived from the beat to beat arterial pressure wave. Stroke volume (SV) was analyzed by the Modelflow (Bogert and van Lieshout 2005). Cardiac output (CO) was calculated as the product of HR and SV and total peripheral resistance (TPR) by dividing mean arterial pressure (MAP) by CO.

To study heart rate variability (HRV), R-R intervals were obtained from a standard ECG. 


\section{Cardiovascular autonomic nervous activity evaluation}

Firstly, baseline autonomic nervous profile of the subjects were evaluated thanks to heart rate (HRV) and blood pressure (BPV) variability analyses performed on a time series of 256 cycles selected during the baseline period, according to standard recommendations (Task Force 1996). The corresponding hemodynamic data are presented in Table 1. Secondly, HRV analysis was performed on a one min-length basis to evaluate the cardiac autonomic nervous response to the CPT. Three different periods were chosen for this evaluation: during the baseline period (corresponding hemodynamic data presented in Table 2), and during the second and third min of CPT. Since maximal discomfort occur in the first min of cold stimulus, the first min was not addressed in the present study (Hilz et al. 2002).

All the R-R intervals, SBP and DBP values used for analyses were edited initially by visual inspection to exclude all the undesirable beats (i.e. to ensure that each analysis for the segment was free of movement artifact and/or sharp transient in the signal due to premature beats) which accounted for less than $1 \%$ in every subjects.

Spectrum analysis was performed with the coarse graining spectral analysis (CGSA) method (Yamamoto and Hughson 1991) that separates and permits simultaneous quantification of the contribution of the harmonic and fractal components of the total spectral power (TP), even when the data length is short (Yamamoto and Hughson 1991). From the harmonic component (HP) the integrated powers $\left(\mathrm{ms}^{2}\right)$ in the low(LF: 0.04-0.15 Hz) and high- frequencies (HF: 0.15-0.50 $\mathrm{Hz}$ ) were computed (Yamamoto and Hughson 1991). The very low frequencies $(0-0.04 \mathrm{~Hz})$ were not addressed in the present study. With HRV, the HF power normalized to the total spectral power (HFnu) was used as an indicator of parasympathetic activity. Despite controversial results, the LF/HF ratio is often used as an indicator of sympathetic activity (Yamamoto and Hughson 1991) and was used with this meaning.

The heart beat times series exhibits pattern of non-linear process and is non-stationary during CPT. To better delineate the cardiac autonomic control, we used the DFA method to quantify the fractal-like scaling properties of the R-R interval data (Peng et al. 1995). The algorithm computes R-R intervals fluctuations in several windows of different sizes and finally creates a log-log curve, the slope of which defines the scaling exponent $\alpha_{1}$. This short-term scaling exponent was computed for small (four to 11 beats) time scales. Details of DFA have been described previously (Peng et al. 1995, Tulppo et al. 2001a,b, 2005).

\section{Spontaneous baroreflex sensitivity}

Sequences of three or more beats in which the SBP and the following R-R interval changed in the same direction (either increasing or decreasing), which reflect the HR response to spontaneous variations in BP, were considered as spontaneous baroreflex (SBR) sequences. A linear regression was calculated for each of these sequences, and an average regression slope was calculated for all such sequences detected during each chosen recording epoch. This slope is considered as depicting the sensitivity of the cardiac SBR ( $\mathrm{ms}^{\mathrm{mmHg}} \mathrm{m}^{-1}$ ) (Bahjaoui-Bouhaddi et al. 2000).

\section{Temperature}

Subjects' skin temperatures of the two hands (middle of the third metacarpus of the palmar side) were measured continuously by means of thermistor surface contact probes [series 400, type 409B, Yellow Springs Instrument (YSI); accuracy $\pm 0.1{ }^{\circ} \mathrm{C}$ ] fixed on the skin with thin, air-permeable, adhesive surgical tape. The probes were applied on the centre of the palmar surface.

\section{Statistical methods}

Standard statistical methods were used for the calculation of mean \pm S.D. Two comparisons were performed. First, the baseline characteristics of the subjects of the CPTi and CPTd groups were compared by Student t-test. Second, the responses to the cold pressor test within each group were evaluated by one-way ANOVA with repeated measures. When appropriate, post-hoc t-tests for paired data with Bonferroni correction were performed. Statistical significance was accepted at the $p<0.05$ level. Statistical analyses were performed using SigmaStat ${ }^{\circledR}$ software (SPSS Inc, Chicago, USA).

\section{Results}

Cold pressor test and measurements were successfully performed in all but one subject, which was removed from the results presented here. In this subject, beat-by-beat blood pressure could not reliably be evaluated during the entire test's duration. Without this subject, the characteristics of the subjects were age $23.7 \pm 3.2$ years, height $180.4 \pm 4.7 \mathrm{~cm}$ and weight $73.3 \pm 6.4$ $\mathrm{kg}$. Based on the HR response to the CPT, 20 subjects 


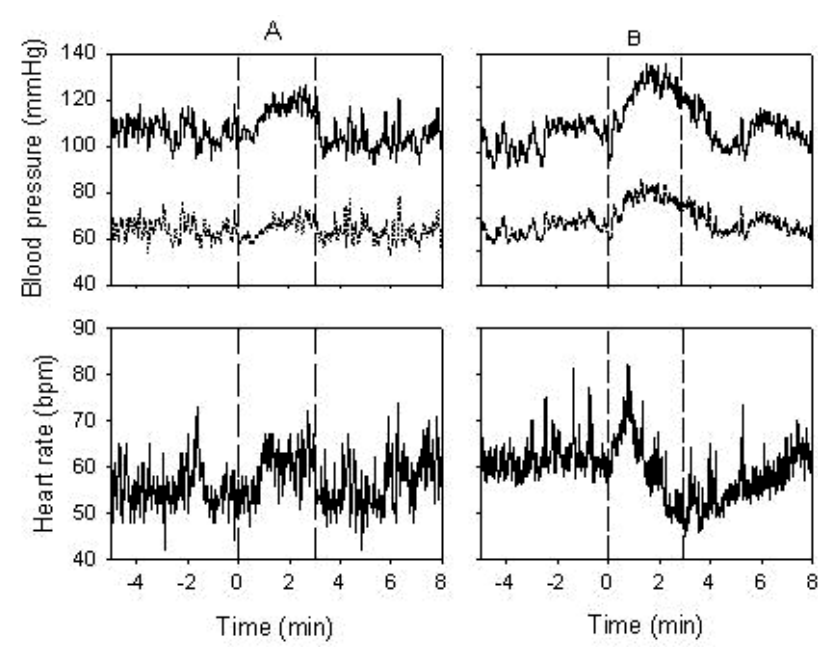

Fig. 1. Changes in blood pressure and heart rate in a representative subject of the CPTi (A) and CPTd (B) groups.

formed the CPTi group and 19 were assigned to the CPTd group.

Baseline characteristics of the two groups.

Anthropometric and hemodynamic data and indexes derived from analyses of HRV and BPV are given in Table 1. At baseline, no significant differences were found between CPTi and CPTd.

\section{Hemodynamic responses during the CPT (Table 2)}

For the two groups, significant increases in BP and TPR were observed during the second and third min of CPT. For CPTi group, the increases between baseline and the third min were $14.7 \pm 10.4 \%, 19.1 \pm 14.6 \%$, and $16.7 \pm 23.9 \%$, for SBP, DBP and TPR, respectively. For the CPTd group, the increases were $12.5 \pm 7.7 \%$, $15.0 \pm 10.2 \%$, and $17.8 \pm 17.0 \%$, for SBP, DBP and TPR, respectively (Table 2). PP also increased, but the increases reached the significant level only for the third $\min (9.6 \pm 10.4 \%$ for CPTi and $9.5 \pm 10.7 \%$ for CPTd).

The palmar surface temperature of the left (immersed) hand decreased significantly for the two groups (Table 2$)$ at the second $(47.6 \pm 22.7 \%$ for CPTi and $55.9 \pm 13.6 \%$ for CPTd) and third $\min (50.1 \pm 24.6 \%$ for CPTi and $58.8 \pm 15.3 \%$ for CPTd). The palmar surface temperature of the right (non-immersed) hand also decreased at the second and third min. However, the decreases were not significant for the CPTi group (1.6 $\pm 4.8 \%$ for the second $\min$ and $1.5 \pm 6.5 \%$ for the third min), while the significant level was achieved for the CPTd group $(1.5 \pm 2.7 \%$ for the second $\mathrm{min}$ and $1.7 \pm 2.3 \%$ for the third $\mathrm{min}$ ).
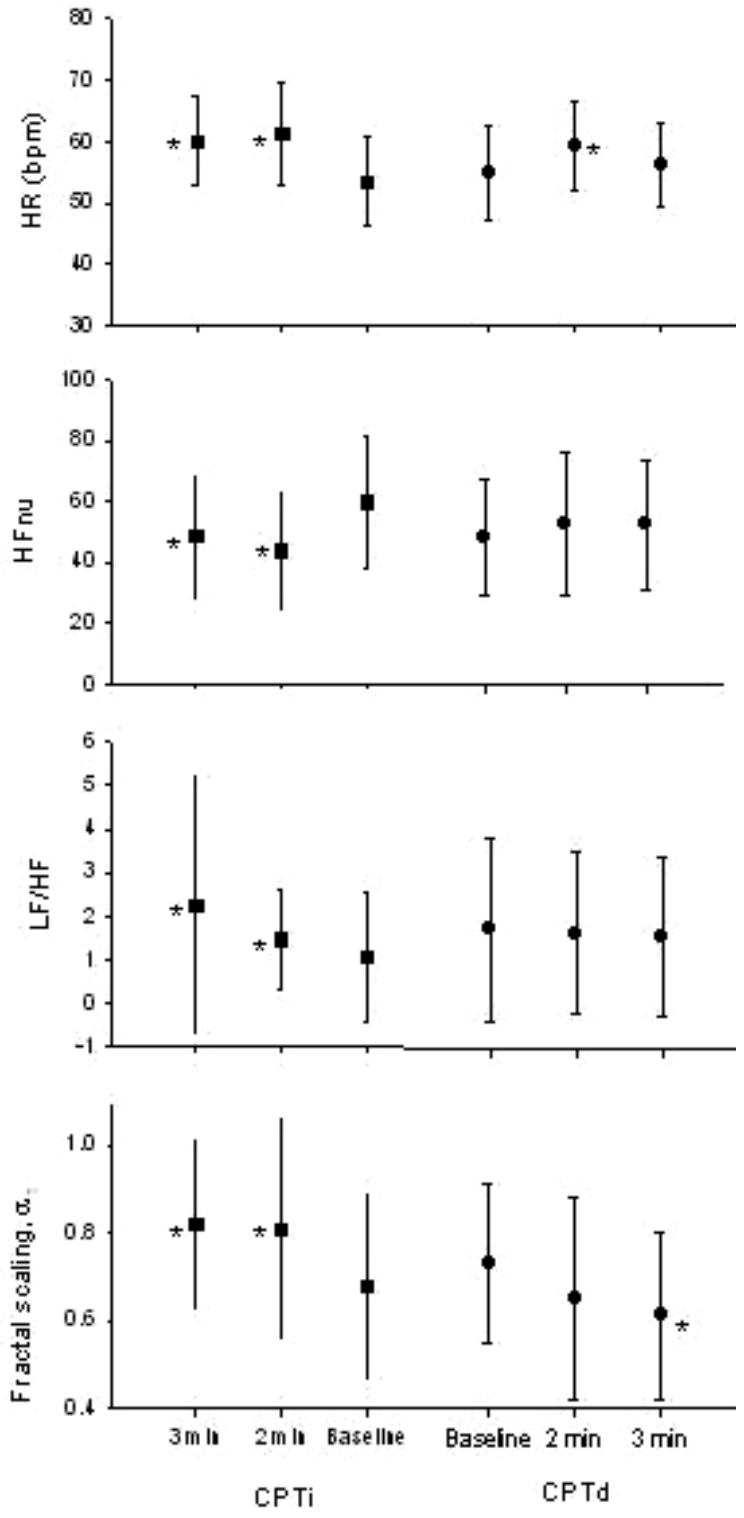

Fig. 2. Changes in heart rate (HR), in normalized high frequency (HFnu) and low- to high frequency ratio (LF/HF) of heart rate variability and in short-term fractal scaling exponent $(\alpha 1)$, measured at baseline and during the second and third min of cold hand immersion in the group with increased (CPTi) or decreased (CPTd) HR response to the cold pressor test (CPT).

\section{Cardiovascular autonomic responses during the CPT}

In the CPTi group, HR was significantly higher at the second and third min compared to baseline (Fig. 2). In absolute values, LF increased from $686 \pm 654 \mathrm{~ms}^{2}$ during baseline to $1617 \pm 1551 \mathrm{~ms}^{2}$ and $1623 \pm 1454 \mathrm{~ms}^{2}$ during the second and third min, without reaching the significant level. HF changed (without statistical differences) from $1045 \pm 945$ to $2040 \pm 2548$ and $1598 \pm 2049 \mathrm{~ms}^{2}$ (second and third min, respectively). This was accompanied by a significant decrease in HFnu and by a significant increase in the $\mathrm{LF} / \mathrm{HF}$ ratio and the scaling exponent $\alpha_{1}$ (Fig. 2). The slope of SBR changed 
Table 1. Anthropometric and baseline hemodynamic and autonomic status of the subjects.

\begin{tabular}{|c|c|c|c|c|}
\hline & & & $\begin{array}{l}\text { CPTi } \\
n=20\end{array}$ & $\begin{array}{c}\text { CPTd } \\
\mathbf{n}=19\end{array}$ \\
\hline \multirow{3}{*}{ Anthropometric data } & Age & years & $23.0 \pm 3.4$ & $24.4 \pm 3.0$ \\
\hline & Height & $\mathrm{cm}$ & $179.9 \pm 4.6$ & $180.9 \pm 4.9$ \\
\hline & Weight & $\mathrm{kg}$ & $73.3 \pm 7.7$ & $73.3 \pm 4.9$ \\
\hline \multirow{7}{*}{ Hemodynamic data } & HR & bpm & $53.3 \pm 6.9$ & $54.2 \pm 7.2$ \\
\hline & SBP & $\mathrm{mmHg}$ & $126.1 \pm 10.2$ & $126.5 \pm$ \\
\hline & DBP & $\mathrm{mmHg}$ & $70.3 \pm 5.5$ & $70.2 \pm$ \\
\hline & PP & $\mathrm{mmHg}$ & $55.8 \pm 8.9$ & $56.3 \pm$ \\
\hline & SV & $\mathrm{ml}$ & $101.1 \pm 13.9$ & $99.6 \pm 11.9$ \\
\hline & $\mathrm{CO}$ & $1 . \min ^{-1}$ & $5.4 \pm 0.9$ & $5.4 \pm 1.1$ \\
\hline & TPR & (PRU) & $1.02 \pm 0.23$ & $1.03 \pm 0.18$ \\
\hline \multirow{5}{*}{ Linear HRV analysis } & Total Power & $\mathrm{ms}^{2}$ & $5027 \pm 3902$ & $4450 \pm 3968$ \\
\hline & LF & $\mathrm{ms}^{2}$ & $479 \pm 547$ & $407 \pm 459$ \\
\hline & $\mathrm{HF}$ & $\mathrm{ms}^{2}$ & $515 \pm 552$ & $677 \pm 1302$ \\
\hline & HFnu & n.u. & $0.11 \pm 0.10$ & $0.13 \pm 0.12$ \\
\hline & $\mathrm{LF} / \mathrm{HF}$ & & $3.28 \pm 5.93$ & $2.48 \pm 4.30$ \\
\hline \multirow{3}{*}{ Linear BPV analysis } & Total Power & $\mathrm{ms}^{2}$ & $24.4 \pm 19.6$ & $20.0 \pm 18.3$ \\
\hline & LF & $\mathrm{ms}^{2}$ & $2.9 \pm 1.8$ & $2.6 \pm 2.5$ \\
\hline & LFnu & $\mathrm{ms}^{2}$ & $15.8 \pm 18.9$ & $19.0 \pm 42.0$ \\
\hline Baroreflex sensitivity & & ms.mmHg ${ }^{-1}$ & $24.6 \pm 10.3$ & $29.0 \pm 9.8$ \\
\hline Non-linear HRV analysis & $\alpha 1$ & & $0.68 \pm 0.21$ & $0.73 \pm 0.18$ \\
\hline
\end{tabular}

CPTi and CPTd - group of subjects with increased or decreased heart rate after the initial increase during the cold pressor test. HR - heart rate; SBP and DBP - systolic and diastolic blood pressure; PP - pulse pressure; SV - stroke volume; CO - cardiac output; TPR - total peripheral resistance. LF - low frequency; HF - high frequency; nu - normalized units; $\alpha 1$ - short-term fractal exponent.

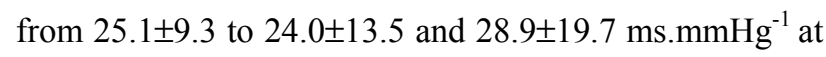
the second and third min (no significant differences).

In the CPTd group, HR was increased during the second min of the CPT ( $p<0.05$ compared to baseline and the third $\mathrm{min}$ ) and decreased thereafter to reach the baseline value at the end of the CPT. LF increased (nonsignificantly) from $701 \pm 654 \mathrm{~ms}^{2}$ during baseline to $1155 \pm 915 \mathrm{~ms}^{2}$ and $1033 \pm 792 \mathrm{~ms}^{2}$ during the second and third min. HF increased significantly from $720 \pm 732$ to $1530 \pm 1441$ and $1630 \pm 2657 \mathrm{~ms}^{2}$ at the second and third min. This was accompanied by a trend for an increase in HFnu and for decrease in the LF/HF ratio, but without reaching the significant level (Fig. 2). Contrary to the changes observed in the CPTi group, the scaling exponent $\alpha_{1}$ decreased significantly during the second minute and further during the third minute (Fig. 2). The slope of SBR changed from $25.2 \pm 5.8$ to $23.1 \pm 8.8$ and $21.2 \pm 11.9$ ms.mmHg ${ }^{-1}$ at the second and third min (no significant differences).

\section{Discussion}

In healthy human subjects, CPT triggers an increase in BP (Victor et al. 1987, Fagius et al. 1989, Stančák et al. 1996, Jauregui-Renaud et al. 2001, Cui et al. 2002). This may be due to an increased CO during the initial period of the test with little increase in muscle sympathetic nerve activity, while an increase in this activity elevates peripheral resistances in the later period (Victor et al. 1987, Yamamoto et al. 1992). Pulse pressure also increases, mainly at the end of the test (Stančák et al. 1996). The results of the present study are 
Table 2. Hemodynamic changes during the cold pressor test.

\begin{tabular}{|c|c|c|c|c|c|c|c|c|c|c|c|c|}
\hline & & & \multicolumn{2}{|c|}{ First min - Baseline } & \multicolumn{3}{|c|}{ Second min } & \multicolumn{5}{|c|}{ Third min } \\
\hline & $S B P$ & $m m H g$ & 125.8 & $\pm \quad 10.3$ & 143.9 & \pm & 20.6 & $*$ & 145.0 & \pm & 22.8 & $*$ \\
\hline & $D B P$ & $m m H g$ & 70.1 & $\pm \quad 5.5$ & 84.0 & \pm & 11.3 & $*$ & 83.5 & \pm & 11.9 & $*$ \\
\hline & $P P$ & $m m H g$ & 55.7 & \pm 9.3 & 59.9 & \pm & 14.2 & & 61.4 & \pm & 14.7 & $*$ \\
\hline$C P T i$ & $S V$ & $m l$ & 101.4 & $\pm \quad 13.8$ & 94.4 & \pm & 19.3 & & 97.6 & \pm & 15.6 & $* \S$ \\
\hline \multirow[t]{8}{*}{$n=20$} & $\mathrm{CO}$ & l. $\min ^{-1}$ & 5.4 & $\pm \quad 0.9$ & 5.7 & \pm & 1.4 & & 5.6 & \pm & 1.0 & \\
\hline & $T P R$ & $(P R U)$ & 1.02 & $\pm \quad 0.21$ & 1.18 & \pm & 0.34 & * & 1.18 & \pm & 0.29 & $*$ \\
\hline & $T^{\circ}$ left hand & ${ }^{\circ} \mathrm{C}$ & 33.0 & $\pm \quad 1.4$ & 17.0 & \pm & 7.2 & * & 16.2 & \pm & 7.9 & $*$ \\
\hline & $T^{\circ}$ right hand & ${ }^{\circ} \mathrm{C}$ & 32.0 & $\pm \quad 1.1$ & 31.5 & \pm & 2.1 & & 31.3 & \pm & 2.4 & \\
\hline & & & \multicolumn{2}{|c|}{ First min - Baseline } & \multicolumn{3}{|c|}{ Second min } & \multicolumn{5}{|c|}{ Third min } \\
\hline & $S B P$ & $m m H g$ & 126.5 & $\pm \quad 7.5$ & 140.9 & \pm & 15.0 & $*$ & 141.9 & \pm & 13.7 & $*$ \\
\hline & $D B P$ & $m m H g$ & 71.6 & $\pm \quad 5.3$ & 84.4 & \pm & 6.3 & $*$ & 82.0 & \pm & 7.3 & $*$ \\
\hline & $P P$ & $m m H g$ & 54.9 & $\pm \quad 6.7$ & 56.5 & \pm & 11.4 & & 59.9 & \pm & 9.8 & $* \S$ \\
\hline CPTd & $S V$ & $m l$ & 99.7 & $\pm \quad 10.9$ & 93.7 & \pm & 16.8 & & 99.4 & \pm & 14.7 & \\
\hline$n=19$ & $\mathrm{CO}$ & l. $\mathrm{min}^{-1}$ & 5.5 & $\pm \quad 1.1$ & 5.5 & \pm & 1.0 & & 5.5 & \pm & 0.9 & \\
\hline & $T P R$ & $(P R U)$ & 1.03 & $\pm \quad 0.20$ & 1.20 & \pm & 0.20 & $*$ & 1.19 & \pm & 0.27 & $*$ \\
\hline & $T^{\circ}$ left hand & ${ }^{\circ} \mathrm{C}$ & 32.3 & $\pm \quad 1.2$ & 14.3 & \pm & 4.6 & * & 13.4 & \pm & 5.1 & $*$ \\
\hline & $T^{\circ}$ right hand & ${ }^{\circ} \mathrm{C}$ & 31.7 & $\pm \quad 1.1$ & 31.2 & \pm & 1.3 & * & 31.1 & \pm & 1.3 & $*$ \\
\hline
\end{tabular}

CPTi and CPTd = group of subjects with increased or decreased heart rate after the initial increased during the cold pressor test. SBP and DBP - systolic and diastolic blood pressure; PP - pulse pressure; SV - stroke volume; CO - cardiac output; TPR - total peripheral resistance. * significantly different from baseline; § significantly different from the second min of the cold pressor test $(p<0.05)$.

in accordance with these observations, whatever the group concerned (Table 2).

The increased $\mathrm{CO}$ is mainly due to changes in HR since SV appears unaltered (Dishman et al. 2003). A maintained HR elevation was found throughout CPT compared to baseline (LeBlanc et al. 1975, Shibahara et al. 1996, Jauregui-Renaud et al. 2001). However, a lot of studies also reported a marked increase in HR followed by a slow decrease (Victor et al. 1987, Stančák et al. 1996, Sendowski et al. 1997, Cui et al. 2002). This biphasic pattern was observed in about half of the tested subjects in the present study. The decrease in HR is difficult to explain since the CPT was initially thought to induce a general sympathetic activation with no change or a decrease in vagal outflow (Frey et al. 1980a, Dishman et al. 2003, Tulppo et al. 2005, Wirch et al. 2006). That was the aim of this study to precise the autonomic control of HR during CPT.

Non-invasive evaluation of the autonomic control of heart rate in real-life conditions is possible by means of HRV analysis (Task Force 1996). However, the results of studies using this technique during CPT are inconsistent (Weise et al. 1993, Jauregui-Renaud et al. 2001, Dishman et al. 2003, Glenn and Ditto 2004, Tulppo et al. 2005, Wirch et al. 2006). These results, obtained at a group level, may be explained by the fact that both HR and the changes in HRV indexes appeared highly variable on an individual basis (Jauregui-Renaud et al. 2001, Glenn and Ditto 2004). The subjects of the present study were divided in two groups, according to their HR responses to $\mathrm{CPT}$. In the $\mathrm{CPTi}$ group, the spectral analysis results indicated a decreased cardiac vagal outflow (HFnu) and an increased sympathetic activity (LF/HF ratio). In the CPTd group, the spectral analysis revealed opposite changes in cardiac autonomic regulation. The changes in spectral analysis indexes likely correctly depicted the cardiac autonomic control, because they were in accordance with the HR alteration. They also were in accordance with previous studies with similar changes in HR.

A number of studies dealing with HRV have shown that R-R intervals exhibit patterns suggestive of non-linear processes. Parameters arising from non-linear methods have therefore been identified. The short-term 
scaling exponent $\left(\alpha_{1}\right)$ of the detrended fluctuation analysis (DFA), computed for small (four to 11 beats) time scales, is one such parameter (Peng et al. 1995).

Tulppo et al. (2005) observed that $\alpha_{1}$ increased during CPT. In their study, HR, muscle sympathetic nerve activity and LF/HF ratio increased while the HF index of HRV spectral analysis decreased, suggesting a generalized cardiovascular sympathetic involvement. These authors suggested that when physiological changes in autonomic regulation occurred with reciprocal interplay, the fractal correlation of HR dynamics increased (Tulppo et al. 2001a, Tulppo et al. 2005). The results concerning the CTPi group were in accordance with these results. On the other hand, $\alpha_{1}$ decreased when both the sympathetic and vagal activity increased (Tulppo et al. 2005). Such a coactivation was suspected but not verified during CPT (Weise et al. 1993). We observed that $\alpha_{1}$ decreased in the CPTd group. In healthy subjects, the result of cardiac autonomic co-activation is a decreased HR (Levy 1971, Tulppo et al. 2005), which is consistent with our results. The enhancement of vagal outflow during CPT is likely a baroreflex correction to the sustained blood pressure increase in the latter part of the CPT. Indeed, the CPT shifts the baroreflex curve expressing the relationship between heart rate and systolic blood pressure to high blood pressures, but does not alter its sensitivity (Cui et al. 2002). The baroreflex is thus capable of appropriately modulating HR during CPT.

It is unclear why subjects react to CPT with reciprocal changes in cardiac autonomic control while other increased the activity of the two branch of the cardiac autonomic nervous system. A distinct baseline autonomic nervous activity (e.g., enhanced sensitivity of the baroreflex, higher vagal outflow) was suspected in the CPTd group. However, no significant differences were found in the baseline hemodynamic and autonomic characteristics (Table 1) and thus we could not confirm our hypothesis. A significant decrease in the palmar surface temperature of the non-immersed hand during the test was found only in the CPTd group. This suggests a higher sympathetic tone to the skin in this group (Kistler et al. 1998). A different involvement of the pain receptors could be argued since the sensation of pain has been suggested to play a major role in HR regulation during CPT (Victor et al. 1987). However, this was not evaluated in the present study.

\section{Limitations}

We did not impose the breathing pattern. The subjects spontaneously adapted their tidal volume and breathing frequency but they were encouraged not to change their breathing pattern. Despite reported controversial results, it has been shown that the amplitude of respiratory-related heart rate oscillations increases at a given respiratory rate as the tidal volume increases (Saul et al. 1989). During a cold pressor test, tidal volume and minute ventilation usually increases (Wirch et al. 2006). In the present study, the respiratory-related heart rate oscillations did not change (CPTd) or decreased during CPT, i.e. were opposite to what would be expected from the changes observed in the ventilatory pattern (Saul et al. 1989). Thus the effect of ventilation was considered minimal. Nevertheless, we can not rule out a potential flaw due to ventilation in our results.

\section{Implications}

CPT has been used for the diagnosis of cardiovascular reactivity in normotensive and hypertensive subjects and the responses to CPT may help to identify normotensive candidates at future risk of suffering from hypertensive disease. Most of the time, the pressor response is based on the BP changes, with little or no attention on the HR alteration. In the present study, the mean changes in BP were similar in the two groups. However, half of the subjects reacted with sign of cardiac autonomic co-activation that decrease $\alpha_{1}$. Fractal organization is flexible, and breakdown of this scaleinvariance (self-similarity) may lead to a more rigid and less adaptable system. A decrease in $\alpha_{1}$ has indeed been observed in various disease states or with advancing age, and appeared as the most potent HRV indicator of a facilitated spontaneous onset of fibrillation (Vikman et al. 1999). A breakdown in the short-term fractal organization in human HR dynamics during CPT could have potential clinical implication, but this had to be verified with further studies.

\section{Conclusions}

In the present study, we used both linear and non-linear method of heart rate variability analysis to study the autonomic control of heart rate during the cold pressor test (CPT). It was found that in half of the tested subjects, reciprocal changes in cardiac autonomic regulation induced a sustained increased in HR. In the other subjects, CPT induced a decrease in HR after an initial increase, likely due to the co-activation of vagal and sympathetic outflow at the heart level which was 
highlighted by a change in HR dynamics from fractal toward more random HR organization.

\section{Conflict of Interest}

There is no conflict of interest.

\section{Acknowledgements}

The authors wish to thank the subjects for their time and cooperation. This work was funded by grants from the French Ministry of National Education, of Research and of Technology (UPRES EA3920).

\section{References}

BAHJAOUI-BOUHADDI M, CAPPELLE S, HENRIET MT, DUMOULIN G, WOLF JP, REGNARD J: Graded vascular autonomic control versus discontinuous cardiac control during gradual upright tilt. J Auton Nerv Syst 79: 149-155, 2000.

BOGERT LW, VAN LIESHOUT JJ: Non-invasive pulsatile arterial pressure and stroke volume changes from the human finger. Exp Physiol 90: 437-446, 2005.

CUI J, WILSON TE, CRANDALL CG: Baroreflex modulation of muscle sympathetic nerve activity during cold pressor test in humans. Am J Physiol 282: H1717-H1723, 2002.

DISHMAN RK, NAKAMURA Y, JACKSON EM, RAY CA: Blood pressure and muscle sympathetic nerve activity during cold pressor stress: fitness and gender. Psychophysiology 40: 370-380, 2003.

FAGIUS J, KARHUVAARA S, SUNDLOF G: The cold pressor test: effects on sympathetic nerve activity in human muscle and skin nerve fascicles. Acta Physiol Scand 137: 325-334, 1989.

FREY MA, SELM EA, WALTHER JW, Jr.: Reflex cardiovascular responses to cold exposure of the face or foot. Jpn Heart J 21: 665-679, 1980a.

FREY MA, SIERVOGEL RM, SELM EA, KEZDI P: Cardiovascular response to cooling of limbs determined by noninvasive methods. Eur J Appl Physiol Occup Physiol 44: 67-75, $1980 \mathrm{~b}$.

FU Q, LEVINE BD, PAWELCZYK JA, ERTL AC, DIEDRICH A, COX JF, ZUCKERMAN JH, RAY CA, SMITH ML, IWASE S, SAITO M, SUGIYAMA Y, MANO T, ZHANG R, IWASAKI K, LANE LD, BUCKEY JC, JR., COOKE WH, ROBERTSON RM, BAISCH FJ, BLOMQVIST CG, ECKBERG DL, ROBERTSON D, BIAGGIONI I: Cardiovascular and sympathetic neural responses to handgrip and cold pressor stimuli in humans before, during and after spaceflight. J Physiol Lond 544: 653-664, 2002.

GLENN D ,DITTO B: Vagal tone biofeedback: respiratory and non-respiratory mediated modulations of vagal tone challenged by cold pressor test. MacGill J Med 7: 2004.

HILZ MJ, AXELROD FB, BRAESKE K, STEMPER B: Cold pressor test demonstrates residual sympathetic cardiovascular activation in familial dysautonomia. J Neurol Sci 196: 81-89, 2002.

JAUREGUI-RENAUD K, HERMOSILLO AG, MARQUEZ MF, RAMOS-AGUILAR F, HERNANDEZGORIBAR M, CARDENAS M: Repeatability of heart rate variability during simple cardiovascular reflex tests on healthy subjects. Arch Med Res 32: 21-26, 2001.

KISTLER A, MARIAUZOULS C, VON BERLEPSCH K: Fingertip temperature as an indicator for sympathetic responses. Int J Psychophysiol 29: 35-41, 1998.

LEBLANC J, DULAC S, COTE J, GIRARD B: Autonomic nervous system and adaptation to cold in man. J Appl Physiol 39: 181-186, 1975.

LEVY MN: Sympathetic-parasympathetic interactions in the heart. Circ Res 29: 437-445, 1971.

PARATI G, CASADEI R, GROPPELLI A, DI RIENZO M, MANCIA G: Comparison of finger and intra-arterial blood pressure monitoring at rest and during laboratory testing. Hypertension 13: 647-655, 1989.

PENG CK, HAVLIN S, STANLEY HE, GOLDBERGER AL: Quantification of scaling exponents and crossover phenomena in nonstationary heartbeat time series. Chaos 5: 82-87, 1995.

SAUL JP, BERGER RD, CHEN MH, COHEN RJ: Transfer function analysis of autonomic regulation. II. Respiratory sinus arrhythmia. Am J Physiol 256: H153-H161, 1989.

SENDOWSKI I, SAVOUREY G, BESNARD Y, BITTEL J: Cold induced vasodilatation and cardiovascular responses in humans during cold water immersion of various upper limb areas. Eur J Appl Physiol Occup Physiol 75: 471-477, 1997. 
SENDOWSKI I, SAVOUREY G, LAUNAY JC, BESNARD Y, COTTET-EMARD JM, PEQUIGNOT JM, BITTEL J: Sympathetic stimulation induced by hand cooling alters cold-induced vasodilatation in humans. Eur $J$ Appl Physiol 81: 303-309, 2000.

SHIBAHARA N, MATSUDA H, UMENO K, SHIMADA Y, ITOH T, TERASAWA K: The responses of skin blood flow, mean arterial pressure and R-R interval induced by cold stimulation with cold wind and ice water. J Auton Nerv Syst 61: 109-115, 1996.

STANČÁK A, JR., YAMAMOTOVÁ A, KULLS IP, SEKYRA IV: Cardiovascular adjustments and pain during repeated cold pressor test. Clin Auton Res 6: 83-89, 1996.

TASK FORCE of the European Society of Cardiology and the North American Society of Pacing and Electrophysiology. Heart rate variability: standards of measurement, physiological interpretation and clinical use. Circulation 93: 1043-1065, 1996.

TULPPO MP, HUGHSON RL, MAKIKALLIO TH, AIRAKSINEN KE, SEPPANEN T, HUIKURI HV: Effects of exercise and passive head-up tilt on fractal and complexity properties of heart rate dynamics. Am J Physiol 280: H1081-H1087, 2001 a.

TULPPO MP, KIVINIEMI AM, HAUTALA AJ, KALLIO M, SEPPANEN T, MAKIKALLIO TH, HUIKURI HV: Physiological background of the loss of fractal heart rate dynamics. Circulation 112: 314-319, 2005.

TULPPO MP, MAKIKALLIO TH, SEPPANEN T, SHOEMAKER K, TUTUNGI E, HUGHSON RL, HUIKURI HV: Effects of pharmacological adrenergic and vagal modulation on fractal heart rate dynamics. Clin Physiol 21: 515-523, 2001b.

VICTOR RG, LEIMBACH WN, JR., SEALS DR, WALLIN BG, MARK AL: Effects of the cold pressor test on muscle sympathetic nerve activity in humans. Hypertension 9: 429-436, 1987.

VIKMAN S, MAKIKALLIO TH, YLI-MAYRY S, PIKKUJAMSA S, KOIVISTO AM, REINIKAINEN P, AIRAKSINEN KE, HUIKURI HV: Altered complexity and correlation properties of R-R interval dynamics before the spontaneous onset of paroxysmal atrial fibrillation. Circulation 100: 2079-2084, 1999.

WEISE F, LAUDE D, GIRARD A, ZITOUN P, SICHE JP, ELGHOZI JL: Effects of the cold pressor test on short-term fluctuations of finger arterial blood pressure and heart rate in normal subjects. Clin Auton Res 3: 303-310, 1993.

WIRCH JL, WOLFE LA, WEISSGERBER TL, DAVIES GA: Cold pressor test protocol to evaluate cardiac autonomic function. Appl Physiol Nutr Metab 31: 235-243, 2006.

YAMAMOTO K, IWASE S, MANO T: Responses of muscle sympathetic nerve activity and cardiac output to the cold pressor test. Jpn J Physiol 42: 239-252, 1992.

YAMAMOTO Y, HUGHSON RL: Coarse-graining spectral analysis: new method for studying heart rate variability. J Appl Physiol 71: 1143-1150, 1991. 